\title{
¿CABE EXPLICAR LA UNIÓN EUROPEA MEDIANTE CONCEPTOS FUNDAMENTALES DE TEORÍA DEL ESTADO?*
}

\author{
RAINER WAHL \\ Catedrático de Derecho Público \\ Universidad de Friburgo
}
SUMARIO
I. Acerca de la brillantez conceptual de la teoría del Estado y de la cuestión de su aprovechamiento en espacios que supe- ran el Estado.
II. La comparación entre el Estado y la inte- gración comunitaria.
III. Tesis acerca de la diferencia de la Unión Europea frente a los clásicos Estados
IV. Democracia y principio democrático: ¿Transferibilidad a la Unión Europea o es- pecífica y singular característica de la UE?
V. Reflexión final.

\section{ACERCA DE LA BRILLANTEZ CONCEPTUAL DE LA TEORÍA DEL ESTADO Y DE LA CUESTIÓN DE SU APROVECHAMIENTO EN ESPACIOS QUE SUPERAN EL ESTADO}

\section{I.1. El acreditado y tradicional vínculo del Derecho Público con la TEORÍA DEL ESTADO Y LA FILOSOFÍA DEL ESTADO}

La ciencia jurídica sobre el Estado y el orden político siempre ha vinculado en Alemania dogmática y teoría, interpretación del Derecho positivo y reflexión

* N. del T.: El texto original "Erklären staatstheoretische Leibegriffe die Europäische Union" ha sido publicado en el libro conjunto editado por Horst Dreier, Rechts - und staatstheoretische 
en el contexto de la historia de las ideas políticas. La importancia de los enfoques positivistas y superadores del positivismo ha sido oscilante; las consideraciones sobre la Constitución fluctuaron frecuentemente entre teoría constitucional y positivismo cautivo de la jurisprudencia del Tribunal Constitucional ${ }^{1}$. Antes de que se supere este positivismo jurisprudencial del Tribunal Constitucional mediante una glosa de alcance europeo aplicada al Tribunal de Justicia ${ }^{2}$ parece necesario recordar esta fructífera complementariedad entre dogmática y teoría. Los nuevos planteamientos teóricos nunca son tan necesarios como cuando se atisban innovaciones. $\mathrm{Y}$ es que no basta entonces la mera actualización del conocimiento, no cabe seguir conformándose con lo acreditado. Desde la segunda mitad del siglo xx hay en el Derecho Público novedades más que sobradas. Como contemporáneo hay motivo suficiente para llegar a la conclusión de que nunca ha habido tanta innovación y cambio. La enorme juridificación vinculada a la victoriosa marcha de ambiciosas Constituciones y a la profundización procurada por la jurisdicción constitucional ${ }^{3}$ significó un gran reto para el Derecho Público, que con ello devino en una combinación de la ciencia del Estado y del Derecho Constitucional. Los trascendentales procesos de europeización e internacionalización han convertido al Derecho público, entendido como public law para las esferas nacional, europea e internacional, en una gran obra en construcción, que precisa de forma apremiante del abastecimiento de la reflexión teórica y del pensamiento filosófico.

El Derecho público alemán es por su tradición un ejemplo de la estrecha y duradera conexión entre el Derecho estatal y la ciencia político-filosófica. El pensamiento y la obra de Hasso Hofmann son un claro ejemplo, contemporáneo y valioso, de esta fructífera senda del desarrollo científico ${ }^{4}$. El Derecho Público, que mira tanto dentro del Estado como fuera de él, debe encontrar una conexión íntima, contínua y duradera entre el Derecho positivo construido con arreglo a exigencias sistemáticas y dogmáticas y las disciplinas en las que se

Schlüsselbegriffe: Legitimität - Representation - Freibeit. Symposion für Hasso Hofmann zum 70 Geburtstag, Berlín: Duncker \& Humblot, 2005. Versión al castellano de Jorge Alguacil GonzálezAurioles.

1 BERNHARD SCHLINK, "Die Enttrhonung der Staatsrechtswissenschaft durch die Verfassungsgerichtsbarkeit", en Der Staat 28 (1989), p. 161.

2 Especialmente llamativo es el "encuentro" de la sistemática tradicional alcanzada por el Derecho alemán con el derecho judicial o derecho del caso propio del Ordenamiento Comunitario. De forma casi por completo contraria, en el Derecho europeo cada problema se resuelve no a partir de las normas y de sus propias pecularidades, sino exclusivamente a partir de la cadena de decisiones del Tribunal de Justicia. Sin embargo, el Derecho es siempre más que lo que expresan los tribunales en sus decisiones. El que la ciencia jurídica en sus - naturalmente no vinculantes - interpretaciones pueda contribuir con una cuota significativa al reconocimiento y la interpretación del Derecho es una experiencia y también riqueza que el Derecho alemán debe y puede insuflar en el Derecho comunitario, proviniente de diferentes fuentes.

3 RAINER WAHL, Das Bundesverfassungsgericht im europäischen und internationalem Umfeld, en Verfassungsstaat, Europäisierung, Internationalisierung, 2003, p. 254.

4 Esto se encuentra documentado especialmente en dos obras recopilatorias suyas, Recht-Politik-Verfassung. Studien zur Geschichte der politischen Philosophie, 1986; Verfassungsrechtliche Perspektiven. Aufsätze aus den Jahren 1980 bis 1994, 1995. 
fundamenta. El Derecho europeo y el Derecho internacional no debieran ser abandonados a su suerte, justo ahora que cuentan con un caudal de normas en expansión.

\section{I.2. LA NECESIDAD DE CONCEPTOS NO CENTRADOS EN EL ESTADO}

Es necesario por tanto acuñar conceptos nuevos. Así como antes la teoría general del Estado y la ciencia política pertenecían al Derecho político del Estado**, el Derecho europeo debe completarse y fundarse como disciplina jurídico-positiva-solo cabe plantearse cómo, a través de qué disciplinas y por medio de qué conceptos. Se podría añadir algo así como una teoría de Europa, una ciencia de Europa o una teoría constitucional europea. De forma expresiva, muchos digno de mención es Peter Häberle 5 - postulan desarrollar, en vez de una ciencia estatal, una ciencia constitucional europea, que debería liberarse de supuestas analogías con los modelos del Estado nacional, pero que debería aprovechar su amplia reserva teórica y la riqueza de sus experiencias ${ }^{6}$. Y un paso más supone plantearse a través de qué conceptos dogmáticos debe completarse y fundarse teóricamente el clásico Derecho Internacional — quizá a través de una teoría jurídica mundial o más bien de una teoría constitucional universal para la denominada Comunidad mundial de Estados constitucionales ${ }^{7}$.

¿Se debe renunciar, como propone Brun-Otto Bryde ${ }^{8}$, a todo el conjunto de principios constitucionales que contienen la partícula conceptual "Estado", como Estado de Derecho, Estado social, Estado federal justo a causa de tal elemento limitador y en su lugar preferir expresiones como constitucionalismo, rule of law o justicia social. Esto podría significar, sin embargo, una renuncia excesiva al propio idioma alemán y al pensamiento ligado a él***. Y es que, por más que debamos poner en relación los conceptos alemanes tradicionales con

** N del T: En este caso traducimos así el término Staatsrecht, cuya versión al castellano resulta siempre complicada: ni entre nosotros es identificable el alcance de un "Derecho del Estado", ni nuestro viejo "Derecho Político" agota, pese a sus difusos márgenes, cuanto en Alemania se comprende bajo el mencionado término.

5 PETER HÄBERLE, Europäische Verfassungslehre, 2 ed, 2004. El pensamiento de Häberle es valorado por Markus Kotzur, "Wechselwirkungen zwischen Europäischer Verfassungs- und Völkerrechtslehre,, en Homenaje a Peter Häberle, 2004, pp. 289 ss. Häberle nunca reduce con ello lo específicamente nuevo y propio de la Constitución de Europa a una supuesta analogía con el modelo nacional estatal.

6 Casi todas las nuevas publicaciones, sobre todo las relativas al proyecto de Constitución, se ocupan del tema de la (no) ejemplaridad de los conceptos jurídico-estatales para la UE, p. ej. Las jornadas editadas bajo el título "Vom Vertrag zur EU-Verfassung? - Der Konventsentwurf in der Analyse, EuGRZ 2004, P. 541 ss, ahí p. Ej. CHRISTIAN CALLIES/MATTHIAS RUFFERT, Vom Vertrag zur Verfassung?, EuGRZ 2004, P. 542, 544.

7 Sobre el particular, M. Morlok (ed), Die Welt des Verfassungsstaates, 2001

8 BRUN-OTTO BRYDE, "Konstitutionalisierung des Völkerrechts und Internationalisierung des Verfassungsrechts", Der Staat 42 (2003), pp. 61 ss.

***: N del T: En alemán, como es conocido, las locuciones mencionadas se expresan con una sola palabra en cuya composición se integra la partícula —Staat: Rechtstaat, Sozialstaat, Bundestaat. 
otros conceptos y con las construcciones conceptuales de otros ordenamientos jurídicos (y viceversa) seguirá siendo necesario que tengamos claro que el principio del Estado de Derecho y el rule of law no significan lo mismo. Se precisa más bien de la conexión creativa de estos conceptos básicos y no sencillamente de la sustitución del concepto nacional por otro concepto por lo demás también nacional. El idioma inglés por sí sólo no llega a elevar el pensamiento a un nivel supranacional o internacional.

Alli donde se ofrecen tantos conceptos, surge pronto la sospecha de que carecemos en realidad de los conceptos adecuados a partir de los cuales se alcanza comprensión. Los conceptos no son naturalmente todo, pero sin conceptos difícilmente cabe alcanzar un entendimiento suficiente. El problema básico del Derecho Público, (oculto o abiertamente discutido) radica en que se mueve cada vez más fuera de los habituales ámbitos del Estado conocidos desde hace siglos, mas para ese viaje exploratorio dispone sin embargo sólo básicamente de los conceptos centrados en el Estado. Cabe aprovechar los conceptos descriptivos, como Staatenverbund" (liga de Estados); no se logra con ello la impresión, sin embargo, de haber dado con el concepto idóneo.

Además, hemos de considerar también la cuestión del criterio, de las ideas o principios. ¿Es posible una transferencia directa desde el ámbito conceptual nacional o no?? ¿Son nuestros conceptos constitucionales y democráticos, por mor de su larga tradición, conceptos generales, que pueden servir para todas las formas y niveles del poder político, o se limita su validez al ámbito de los Estados nacionales? Se impone una cuestión adicional: detrás de los problemas conceptuales se encuentra en realidad la cuestión de las particularidades y las diferencias entre las tres unidades de la vida política, esto es, los Estados, la Unión Europea y las organizaciones internacionales.

\section{LA COMPARACIÓN ENTRE EL ESTADO Y LA INTEGRACIÓN COMUNITARIA}

Las siguientes reflexiones versan sobre una comparación. Como es conocido, comparar significa poner en relación cosas no iguales. Se habla tanto de una comparación entre los estados individuales y la Unión Europea, justamente porque son diferentes en numerosos aspectos. La cuestión de hasta qué extremo son diferentes es sin embargo una pregunta abierta.

\section{II.1. LA TESIS DE LA REPETICIÓN}

Analizaré para la comparación propuesta una idea que pocas veces ha sido articulada pero que a menudo subyace inconscientemente a las exposiciones y

9 ARMIN VON BOGDANDY, "Zur Übertragbarkeit staatsrechtlicher Figuren auf die Europäische Union. Vom Nutzen der Gestaltidee supranationaler Föderalismus anhand des Demokratieprinzips", en Festschrift für Peter Badura, 2004, pp. 1033 ss. 
que quisiera denominar la tesis de la repetición. Argumenta del siguiente modo: bien pueden existir diferencias entre los Estados y la Unión Europea; hay sin embargo en general algo semejante a una igualdad estructural entre ambos elementos. ¿O acaso debería darse en la Unión Europea realmente otra democracia, otro concepto de pertenencia u otro concepto de Estado de Derecho o de protección de los derechos fundamentales? Al menos la cláusula de garantía estructural del artículo 23 I LF y su correspondiente en el artículo 6 TUE se encaminan en esta dirección de la (necesaria) similitud estructural ${ }^{10}$. Prevén para los elementos constitucionales más importantes de la Unión Europea una suerte de igualdad, paralelismo o repetición del nivel estatal.

\section{II.2. ¿LA INTEGRACIÓN EUROPEA COMO REPETICIÓN DE LA UNIFICACIÓN ALEMANA DEL SIGLO XIX?}

La idea de partida de la repetición goza en Alemania de una particular aceptación, pues ya en el siglo xix hubo un proceso de unificación, la unidad nacional, que al menos se pretende comparar con el desarrollo europeo ${ }^{11}$. A continuación jugaremos a experimentar con ideas para mostrar que cabe extraer enseñanzas de la diferenciación entre ambos acontecimientos. La unificación alemana del siglo XIX, a primera vista comparable con el actual desarrollo europeo, ocurrió mediante la reunión de varios Estados alemanes preexistentes $^{12}$. Se produjo una construcción federal mediante la cual los anteriores Estados individuales se integraron en un sistema de poder a dos niveles. Sobre los antiguos Estados se construyó una nueva cubierta común, o mejor dicho, todo un nuevo edificio que muy pronto se evidenció como vigoroso. En Alemania se puede encontrar ulteriormente una igualdad estructural entre los anteriores Estados individuales y la nueva gran unidad, pues por ejemplo ambos disponían de la monarquía como forma de Estado.

Para la reflexión ulterior, en conexión con lo que acabamos de decir, el elemento decisivo es el gran dinamismo que el proceso de unificación tuvo en los ámbitos estatal y social. En relación con la unidad nacional en Alemania, y a pesar del arraigo de los ciudadanos individuales en los primigenios Estados, a la larga no cupo dudar de que lo primario y decisivo en la nueva constelación ge-

10 El deseo de esta fórmula de referencia recíproca puede comprenderse fácilmente: cuando una Constitución nacional y después también el TUE consideran algunos principios importantes e irrenunciables para el ordenamiento interno, entonces no pueden renunciar a ellos cuando se asocian en una unidad superior.

11 En el desarrollo siguiente se pretende realizar una comparación limitada, sin presuponer o pretender una teoría general. No se plantea la unidad nacional del siglo xIx ni la idea nacional como un ejemplo a imitar. Se trata tan sólo de identificar las fuerzas actuantes en la unificación de entonces y de cuestionarse si hoy obran fuerzas similares y cómo puede comprenderse una constelación que no puede recurrir a fuerzas de esta magnitud.

12 Valorar la pertinencia de estas reflexiones también para el caso italiano precisaría de una investigación detallada; la unificación de Italia se desarrolló de modo diferente a la alemana. 
neral era que las personas y los pueblos de los Estados separados querían la unidad, convertirse en un Estado nacional. Los alemanes dispersos entonces en los numerosos Estados diferentes ${ }^{13}$ se sentían como un pueblo y querían en consecuencia vivir en un Estado ${ }^{14}$. La creación de la nación y del Estado nacional procuró gran vigor al Estado y al poder estatal (como suma del potencial del que el Estado dispone). La identificación y la predisposición a incorporarse, o a dejarse unir, crecieron considerablemente. El Estado legitimado por la idea nacional era más fuerte que el anterior Estado dinástico. El Estado nacional logró un pueblo políticamente movilizado ${ }^{15}$.

En la Europa de los seis, de los quince y de los actualmente 25 Estados miembros se trataba sin embargo desde un principio de una integración, en primer lugar en el ámbito económico, después en otros ámbitos de la sociedad. Se trataba y se trata de la integración parcial y progresiva, ciertamente con la intención política de lograr una posterior unión política o cualquier forma política de unificación. Lo cierto de todos modos es que en 1958 no existió una unidad política (o un deseo de unidad desde abajo), no se produjo luego de inmediato y tampoco está hoy, después de 46 años, ni mucho menos próxima. El desarrollo de la Unión Europea no se construye ni a partir de impulsos poderosos de un pueblo político unido ni a partir de una inequívoca voluntad de cada uno de los pueblos europeos. Lo determinante ha sido y es la voluntad de las elites políticas y económicas.

La integración es un proceso específico que al principio no construye una unidad política superior (y tampoco lo ha hecho después de 46 años). La comunidad de integración "Unión Europea" es un actor muy efectivo, pero no es el punto de referencia y fuente de un sentimiento de pertenencia y de comunidad. Con la unidad del siglo xIX, determinada por la nación, muy pronto pasó el impulso de desarrollo y el centro político del poder de los Estados individuales a la unidad superior; esto es, la unidad nacional central fue determinante con relativa rapidez. En consecuencia, los Estados hasta entonces individuales soportaron la pérdida de significado porque también las personas que residían en ellos querían la unidad nacional. La pérdida de autonomía y so-

13 Básicamente se sabía quién era alemán y quién no. Al menos estaba claro bajo el segundo impulso dado por Bismarck tras la guerra austíaco-prusiana quién debía pertenecer a la nueva Alemania y quién no.

14 Quizá al principio existían todavía dudas sobre dónde residía el peso de la vida política y sobre todo sobre al sentimiento de las personas: aún orientadas hacia los Estados tradicionales o hacia la nueva unidad nacional. Puede que esta duda pesara largo tiempo en la conciencia de muchos alemanes. La velocidad de identificación de la unidad alemana del siglo XIX, comparada sin embargo con los 46 años que ya dura el proceso de integración europeo y en el que la Unión no se ha convertido todavía en el centro de la experiencia política de las personas, es incomparablemente mayor.

15 Wolfgang Reinhardt describe este proceso en su profundidad en Geschichte der Staatsgewalt, 3 edic. 2002, pp. 440 ss, en la página 441 advierte que la movilización emocional sólo era comparable a la religiosa, como ya había sido apuntado por Alexis de Tocqueville. Esto no tiene como última consecuencia el que en el Estado nacional lo religioso se secularice en lo nacional y lo secular se sacralice (Thomas Nipperdey). 
beranía de los Estados individuales preexistentes fue objetivamente mucho mayor que la que se aprecia hoy día por causa de la Unión Europea. Pero lo decisivo no es la pérdida objetiva, sino la valoración subjetiva del proceso. Los pueblos y los Estados individuales en Alemania valoraban mucho más los beneficios de la unificación. La fuerza que tenía la idea nacional fue responsable de que para los nacionales de los Estados originarios, para Baviera, Hesse, Württemberg, etc, pudiera ser atractivo en primera línea ser alemán, y sólo después bávaro, de Hesse o de Würtemberg.

Justo este proceso es el que no ha tenido lugar en los 46 años de Comunidad Europea. Y esta es la diferencia esencial entre la integración comunitaria y el Estado. En una integración no se desplaza el centro de poder, el centro de identificación hacia la unidad superior, esto es, la Unión Europea. Puesto que en la unificación europea - y sobre esto no existe disputano se dispone de una idea nacional, de una suerte de "nación europea" ${ }^{16}$, le ha faltado al proceso de integración mucho de la fuerza motriz que sí se ha desencadenado en las unificaciones nacionales o en la formación de la nación. La integración europea no es, por lo tanto, la repetición de una formación nacional. Toda vez que esto no es discutido, resulta acertado e importante añadir que se plantea así de nuevo y con resultado incierto la cuestión sobre las fuerzas que empujan y acompañan la integración. Por lo que cabe apreciar hasta ahora, la integración no-nacional ha desarrollado un tempo, una intensidad y una fuerza significativamente menor que los procesos de unificación nacional ${ }^{17}$.

A falta de una verdadera unidad política de origen y sin haberla alcanzado aún, en el proceso europeo se integran sectores concretos y ámbitos sociales específicos mediante la supresión de barreras y la creación de un mercado interior. Este fue el camino del crecimiento en común como proceso sectorial-funcional sin la condición previa de que los europeos en su voluntad política y en sus emociones se hubieran decidido ya por la unidad. En la unificación política nacional podemos decir que el pueblo existe ya para la construcción de la unidad, se precisa "tan sólo" la forma política de la unidad. Acto seguido se desarrollan conjuntamente los sectores concretos y la consecuencia es la integración. Mediante una integración como la europea se precisa de un proceso de más años, un largo tiempo hasta que se consiga una formación de unidad política. El final político está abierto, y por ello también queda abierto que a la integración le vaya a seguir la unidad política.

16 Europa no se ha deslizado hacia lo nacional ni ha devenido en nación. Está al menos abierta la cuestión de si ésto se encuentra en camino.

17 La integración europea se suele diferenciar de los tempranos desarrollos estatal-nacionales frecuentemente aludiendo sólo a que, de modo consciente y exitoso, se habría evitado el nacionalismo en la integración. Quien afirma esto y lo celebra $-\mathrm{y}$ es ciertamente digno de celebración- debe ser realista y ver la otra cara de la moneda: la falta de las fuerzas propias de los Estados nacionales y de la idea de Estado nación (y sabemos lo vigorosas y destructivas que son), supone inicialmente un déficit, a menos que haya otras fuentes productoras de energía. La cuestión debe plantearse por lo tanto de forma positiva: de dónde obtiene la UE sus fuerzas. 


\section{II3. ¿LA INTEGRACIÓN COMO UN MINUS O UN ALIUD FRENTE A LAS CLÁSICAS UNIFICACIONES POLÍTICAS?}

A la vista de las mencionadas diferencias entre la comunidad de integración y el Estado se ha de plantear de nuevo la cuestión de si los principios político-constitucionales realizados en los Estados pueden ser modelo para la Unión Europea $^{18}$. Si no se quieren dar por sentados prejuicios implícitos al reflexionar sobre la Unión Europea y su estructura, debe tenerse clara una alternativa fundamental para el resultado de las consideraciones que se hagan. Lo decisivo, a fin de cuentas, es si se comprende la Unión Europea como un $m i$ nus o un aliud respecto de la unificación política.

La integración puede comprenderse como un minus frente a la unificación si se espera y se desea alcanzar en el futuro, al final, la completa unidad política. En ese sentido se cualifica idealmente a la Unión Europea como un todavía-no-Estado, al Parlamento Europeo como un todavía-no-parlamento. Todo esto queda bajo la premisa de que la Unión Europea al final alcanzará los rasgos definidores del Estado - una precomprensión que cuenta con la valoración contraria tanto de muchos defensores como de los enemigos de la integración europea.

También puede comprenderse la integración como un aliud, es decir, como una alternativa a la unificación política. Entonces se espera que el desarrollo en común se detenga antes de llegar a la unificación política y que la comunidad de integración encuentre un lugar en el ancho espectro de las relaciones interestatales. Este espectro es muy amplio y lo que realmente interesa de los acontecimientos. Sobre todo dispone de mucho espacio entre los polos extremos. Sobre este espectro podría moverse la UE en los próximos años de modo muy considerable. Podría apreciarse crecimiento y desarrollo, sin que por ello la Unión Europea hubiera de quedar colocada inmediatamente al lado de los Estados federales ${ }^{19}$. La comunidad de integración sería entonces un concepto autónomo, no la denominación de una forma previa al Estado (federal).

\section{TESIS ACERCA DE LA DIFERENCIA DE LA UNIÓN EUROPEA FRENTE A LOS CLÁSICOS ESTADOS}

Las próximas reflexiones girarán en torno a la necesidad de comprobar si los conceptos determinantes como democracia, Constitución o Estado de Derecho se encuentran indisolublemente unidos a la constelación nacional o si es-

18 Es cuestionable sin embargo que el patrón estatal se adecue a la forma y al contenido de la UE alcanzable y deseable en el próximo desarrollo. Está extendido el parecer, si es que no es una opinión general, que la UE no es un Estado. En sentido contrario, JÖRN SACK, "Die Staatswerdung Europas - Kaum eine Spur von Stern und Stunde", Der Staat 44 (2005), p. 67. Menor consenso existe acerca de si las estructuras estatales son al menos patrones para la futura formación y si la UE se desarrolla o se debe desarrollar a semejanza de los Estados.

19 CHRISTIAN CALLIES/MATTHIAS RUFFERT (nota 6), p. 545. La estructura y la organización de la UE se mantienen en suspenso entre el Estado y las organizaciones internacionales. 
tán abiertos para una nueva interpretación en la que se pueda introducir y "Comprender" los niveles supra e internacionales; si, por lo tanto, los conceptos hasta ahora propios de la teoría del Estado pueden utilizarse de forma paradójica para las comunidades y unidades no estatales. Es comprensible que este extenso programa solo pueda ser expuesto fragmentariamente, algunos problemas serán tratados mediante el simple enunciado de tesis.

\section{III.1. LA UNIÓN EUROPEA Y EL ESTADO}

La cuestión de si la Unión Europea es o no es un Estado, más exactamente un Estado federal, ha propiciado la más amplia discusión del nuevo Derecho Público $^{20}$. La respuesta depende naturalmente del concepto de Estado que se adopte. El hecho de que haya y deba haber variedad en el concepto de Estado y en la realidad de los Estados es evidente a la vista de las profundas transformaciones que han experimentado los Estados (nacionales) en los últimos cinco siglos. Por eso el Estado no es, a pesar de su condición indiscutible como (el) concepto esencial del desarrollo político durante siglos, una entidad estable, aunque tampoco es un concepto amoldable de cualquier modo. Es llamativo que autores que afirman la cualidad estatal de la Unión Europea ya o a más tardar con la aprobación del Tratado Constitucional, comprendan el concepto de Estado más bien en un sentido generalizador, por ejemplo cuando reducen a esquema la teoría de los tres elementos del Estado y luego la aplican de forma positiva a la Unión Europea ${ }^{21}$. Cuanto más crece la generalización y abstracción del concepto de Estado y más se deshace el lazo que une los elementos del Estado, y con él la compleja constelación nacional ${ }^{22}$, mejor podrá cualificarse a la Unión Europea como un Estado, pero sólo en ese sentido débil en su concep-

20 La discusión sobre si la estatalidad de la Unión Europea ha alcanzado su punto máximo en el debate sobre el Tratado de Mastrique al comienzo de los años noventa y desde entonces se ha desarrollado toda suerte de fórmulas delimitadoras. Entre ellas destaca en la discusión alemana la fórmula adoptada por el Tribunal Constitucional y acuñada por Paul Kirchhof "Staatenverbund": BVerfGE 89, 155 (184); sobre el particular, recientemente de nuevo PAUL KIRCHHOF, "Die rechtliche Struktur der Europäischen Union als Staatenverbund", en A.V BOGDANDY (ed), Europäisches Verfassungsrecht. Theoretische und Dogmatische Grundzüge, 2003, pp. 892 y ss. De la discusión sobre la estatalidad de la UE y la transferibilidad a ella de conceptos jurídico-estatales véase con amplias referencias adicionales, ARMIN VON BOGDANDY, Supranationaler Föderalismus als Wircklichkeit und Idee einer neuen Herrschaftsform, 1999, pp. 29 y ss, y Stand und Entwicklungsperspektiven rechtswissenschaftlicher Konzepte zum europäischer Integration, 2001, pp. 107, 112 ss.

21 Recientemente ha defendido de nuevo JÖRN SACK (nota 18), que el proceso de conversión de la Unión Europea en Estado ya ha tenido lugar hace tiempo, en todo caso con la aceptación de un Tratado Constitucional.

22 Sobre el particular, las observaciones de HASSO HOFMANN, "Zu Entstehung, Entwicklung und Krise des Verfassungsbegriffs", Festschrift für Peter Häberle, 2004, p. 170 nota 52 sobre la "completa y teóricamente ambiciosa obra" de ANNE PETERS, Elemente einer Theorie der Verfassung Europas, 2001: "La obra muestra de forma impresionante cómo el concepto concreto de Constitución, ligado a una época histórica, se desmorona en pedazos aislados y combinables a placer cuando se prescinde del perfil histórico". 
to y contenido. No se atiende con ello, sin embargo, con tal abstracción y con tal método de formación débil de conceptos, a la intención formulada al principio de concebir teóricamente la Unión Europea y profundizar su comprensión en esta perspectiva.

No cabe conformarse por tanto etiquetando la Unión Europea como un Estado como una "liga de estados" (Staatenverbund) o como una liga constitucional (Verfassungsverbund); tampoco es satisfactoria la denominación "construcción sui generis" cuyo carácter confuso resulta de su propio tenor. La simple tesis de que la Unión Europea no es un Estado en el sentido del (posiblemente también mudable) concepto de Estado sólo tiene un carácter negativo, delimitador. Únicamente tendrá sentido cuando sea capaz de dejar atrás la fórmula sui-géneris ${ }^{23}$ y caracterizar positivamente primero los rasgos estructurales de la UE, para posteriormente enunciar una fórmula general para el contenido y la forma de la Unión Europea. Dado que esto último debe ser el segundo paso, las consideraciones siguientes han de concentrarse en el primero. El problema de partida de la idoneidad de los conceptos teóricos clave para explicar las características estructurales y no inmediatamente para la descripción de la estructura general de la Unión Europea está especialmente justificado porque los elementos y principios singulares de la Unión permiten mostrar suficientes particularidades de la Unión Europea y diferenciarla frente a los Estados ${ }^{24}$, de modo que la aceptación de que la UE no es un Estado resulta ser mucho más plausible que su contraria.

\section{III.2. La Unión EUROPEA COMO FEDERACión (BUND)}

La Unión Europea se presenta en primer lugar como un orden federal, para lo cual son característicos especialmente los siguientes elementos.

a) Su futuro se mueve dentro del espectro de los ordenamientos federales, donde la dicotomía "Unión de Estados versus Estado federal" ni actualmente ni tampoco en el futuro ofrece una orientación adecuada. El Estado federal no es ni la única ni la más verosímil orientación.

b) La Unión Europea es una federación y como tal una comunidad política que no puede ser comprendida mediante conceptos centrados en el Estado (unitario) sino que muestra estructuras o características propias de naturaleza federal. Se puede describir por lo tanto como un concepto que está determinado por estructuras propias, no precisamente estatales. Así lo ha expuesto reciente-

23 La fórmula "Sui-géneris" expresa sólo y no es poco, la distancia de la Unión Europea respecto del Estado, pero sólo tiene carácter negativo y no posee la capacidad de aclarar positivamente la situación.

24 Algunas diferencias sólo pueden ser mostradas aquí como lemas: pueblos — pueblo, forma de creación de la Constitución - poder constituyente, Tratado Constitucional —Constitución, competencia universal y competencia de la competencia- atribución limitada de competencias. 
mente Christoph Schönberger ${ }^{25}$. El moderno concepto de federación representa un pensamiento que acumula, operando mediante el recurso a la fórmula "a la vez, también". Así se justifica el hecho de que la federación y los Estados miembros posean, cada uno, una existencia jurídico-política propia. La vinculación realizada en la federación se evidencia en muchas estructuras en forma de adición (tanto una cosa como la otra) y no de exclusión (o bien la una, o la otra): Estado federal o Federación de Estados, Estado u organización internacional, Derecho estatal o Derecho internacional.

El concepto de federación no surge como la anterior tradición de un concepto general débil de "relación de Estados" que se rompe de repente a favor de unos conceptos alternativos Unión de Estados o Estado Federal; se caracteriza por admitir un amplio espectro de vinculaciones y agregados de órdenes federales. El enfoque no se dirige a la disolución del espectro en un dualismo de supuestos conceptos generales que en la realidad están determinados por la historia. En su lugar insiste en que el espectro debe ser comprendido de forma no reductiva y en que la amplia pluralidad, rica en consecuencias, de fórmulas singulares que aparecen en dicho espectro no debe ser entendida como catálogo de "formas intermedias" sino como formas independientes de órdenes federales. Frente a la primera apariencia de que el tema federal es un tema propio de una teoría general (del Estado), se evidencia que todos los órdenes federales, sea el Bund alemán (1815-1866), el imperio como Estado federal, la República Federal como un Estado federal unitario, los Estados Unidos de América, Canadá, Suiza y Austria son en gran medida individualidades histórico-políticas. En esta serie de órdenes federales singulares en el marco del espectro se incluye la Unión Europea. Comprendida como federación, comparte con otros órdenes federales ${ }^{26} \mathrm{ca}-$ racterísticas que proceden de la simultaneidad de la federación y los Estados, por ejemplo el balancearme sobre situaciones de equilibrio de poderes en continua transformación, la lábil limitación de las competencias (y más aun de la efectiva distribución del poder), una situación en suspenso en cuanto al tema de la soberanía, central sólo en los Estados unitarios. Los órdenes federales son descritos precisamente mediante la naturaleza procesal de los desarrollos de las estructuras y de las delimitaciones entre la federación y los Estados miembros.

La reflexión en torno al concepto de federación es fructífera y estimulante porque en primer lugar permite llamar la atención sobre la duplicación, la característica de liga y de estructura a varios niveles de los órdenes federales, y en segundo lugar porque conduce al pensamiento teórico desde las dos unidades separadas hacia el conjunto y por lo tanto requiere integrar en el campo visual la constelación general "Unión Europea más Estados miembros".

25 CHRISTOPH SCHÖNBERGER, "Die Europäische Union als Bund", AöR 129 (2004), pp. 81 ss.

26 Es sin embargo importante hacer notar que no se acepta una ley histórica por la que todas las relaciones tenues se convierten con el tiempo y la mayor parte de las veces con relativa rapidez en un Estado federal. En el mundo de las formaciones políticas no existen tales tipos de leyes. 
c) La Unión Europea es, a diferencia de los conocidos Estados federales, un orden federal en el que la sustancia política no está en la gran unidad sino en las pequeñas, los Estados miembros. Mediante la denominación "sustancia política" se pretende indicar que las ciudadanas y ciudadanos de Europa, cuando tienen interés político y ponen esperanzas en la política, cuando están preparados para comprometerse en la política y consideran la política como algo importante, se acogen a sus Estados miembros y no piensan en la Unión Europea $^{27}$. La consecuencia es que los europeos se sienten en primer lugar ciudadanos de sus Estados y sólo en segundo lugar, si acaso, como ciudadanos de la Unión. Las unidades inferiores son el punto de cristalización para la parte principal de la energía política que aportan las ciudadanas y los ciudadanos en Europa. Su interés se dirige a la política de los Estados miembros. La necesidad de política, de discusión, compromiso y cooperación se satisface de forma preponderante en la unidad inferior. Esto trae causa no sólo del hecho de que el Parlamento Europeo no tenga "plenos" derechos, sino que es tributario del mucho más elemental hecho de que todavía después de 46 años las personas se interesan más por su Estado que por la Unión Europea. Bien parece, o más bien resulta seguro, que las ciudadanas y los ciudadanos no se dejan imponer por las teorías de los profesores de Derecho Político o Europeo qué unidad debe suscitarles más interés ${ }^{28}$.

\section{III.3. LA INUTILIDAD DEL CONCEPTO DE SOBERANÍA}

A la renuncia a una prudente relación con los conceptos centrados en el Estado le corresponde seguidamente el abandono del concepto de soberanía cuando se habla de la relación entre la Unión Europea y los Estados miembros. La afirmación de la soberanía de ambas partes - nos referimos de un lado a la posición de que los Estados nacionales son los dueños de los Tratados y del otro a la de la autonomía de la Comunidad que ha cortado su cordón umbilical con los Estados-no acierta en lo nuevo y característico de la federación. Sólo se puede hablar de soberanía en la Unión Europea en la medida en que ésta está en suspenso y mientras la Unión Europea sea una federación permanecerá en suspenso. El problema aludido mediante la cuestión de la soberanía se resuelve paradójicamente cuando no se plantea esta cuestión. Muchos son tam-

27 El que la Unión Europea determine y acuñe de manera creciente las decisiones políticas en los Estados miembros no es un contra-argumento. La realidad del significado de la UE y la percepción que de ella tienen los ciudadanos son muy divergentes. Esta significativa discrepancia no se debe ver como una pequeña dificultad de los momentos iniciales, fácilmente superable; persiste ya durante los 46 años de la UE.

$28 \mathrm{El}$ que los europeos, así se expresa la tesis, se interesen actualmente en primer lugar por la política nacional puede cambiar. Pero tendrá que cambiar antes de que la Unión Europea alcance una nueva etapa en su desarrollo. El cambio no se producirá porque una teoría así lo exija, sino porque y en la medida que los europeos vean motivos suficientes para tal cambio y lo lleven a cabo. 
bién conscientes de que en el supuesto de que efectivamente se planteara la cuestión de la soberanía en la UE, ésta entrará en una crisis decisiva ${ }^{29} 30$.

El que se haya prescindido en realidad del viejo concepto de la soberanía, aun cuando permanezca en la boca de todos, se evidencia en la fórmula ampliamente usada de soberanía compartida. Describe de forma exacta la situación de que ni la Unión Europea ni los Estados pueden funcionar de forma autónoma. Pero una soberanía dividida ya no es la soberanía que habían concebido en un primer momento Bodino y sus seguidores. Mediante esta denominación de soberanía compartida, este concepto central para toda cuestión que afecte al poder político-social resulta singularmente inofensivo y amansado - un indicio más de que no es el concepto jurídico-político central del orden federal de la Unión Europea.

\section{III.4. El CONCEPTO CONSTITUCIONAL Y LA UNIÓN EUROPEA}

La Constitución de la Unión Europea como una federación y una Comunidad de Integración debe entenderse como un aliud frente a la Constitución de un Estado ${ }^{31}$, pues la Comunidad de Integración y la federación son también diferentes al Estado. Puesto que los conceptos son siempre meros acuerdos intersubjetivos se puede emplear — condicionado al correspondiente acuerdoel término Constitución de igual manera también para la Unión Europea, si bien en este caso probablemente el concepto Tratado constitucional refleja mejor la singularidad y la diferencia con la Constitución de los Estados. Lo característico de la Unión Europea como federación es que las fuentes de legitimación y poder dominantes parten de los Estados hacia la Unión Europea; en cualquier caso, no son más débiles que las que proceden directamente de los pueblos europeos en dirección a la UE. La Unión Europea es un régimen de

29 Para esto hace falta también que en los ordenamientos federales, mientras el desarrollo tenga buen futuro y no se plantee la cuestión de la soberanía o sea contemplada como secundaria, le corresponda a la razón práctica sostener la suspensión y permanecer alejada de teoremas de otros pasados ordenamientos. Se dejan aparte estos teoremas y no nos dejamos seducir por la idea de la soberanía como un concepto de validez general que debe ser aclarado también en el orden federal de forma inequívoca. La pretensión de aclaración conduciría al orden federal en primer lugar a la verdadera crisis.

30 En el contexto teórico (estatal): Jean Bodin es el teórico de la soberanía, pero justo por ello no es el teórico de los órdenes federales.

31 La bibliografía sobre las posibilidades de constitucionalización de la Unión Europea son entretanto inabarcables, vid, DIETER GRIMM, "Braucht Europa eine Verfassung?", JZ 1995, pp. 581 Ss (en particular 586); ERNST-WOLFGANG BÖCKENFÖRDE, Welchen Weg gebt Europa, 1977; GRISTIAN KOENIG, "Ist die Europäische Union verfassungsfähig?", DÖV, 1998, pp. 268 ss; INGOLF PERNICE Y PETER M. HUBER, "Europäisches und nationales Verfassungsrecht", VVDStRL 60 (2001), pp. 148 ss; CHRISTOPH MÖLLERS, "Verfassungsgebende Gewalt - Verfassung - Konstitutionalisierung", en Av Bogdandy (nota 20), pp. 1 ss; Paul Kirchhof (nota 20); MANFRED ZULEEG, "Die Vorzüge der Europäischen Verfassung", p. 931; CHRISTIAN CALLIES/MATTHIAS RUFFERT (nota 6), pp. 541 ss. 
vinculación débil con los ciudadanos; hay una ciudadanía europea, pero no alcanza hasta el momento la fuerza de la pertenencia como nacional a los Estados.

Sería erróneo por lo tanto reprochar también que el Tratado Constitucional de la Unión Europea no es una "verdadera" Constitución o una Constitución en sentido propio. Los conceptos en sí mismos no son verdaderos, tienen un ser propio, son simplemente acuerdos. Reprochar al Tratado Constitucional europeo el que no es una Constitución verdadera o genuina significa simplemente que la Constitución europea no se corresponde con el modelo de las Constituciones clásicas estatales. Pero esta constatación no nos conduce más allá, en el fondo es trivial. Pese a ello, en el contexto constitucional está bien observado ${ }^{32}$ : la Constitución de la Unión Europea sigue sin ser establecida o modificada por sus propios órganos de forma autónoma, por el Consejo de Ministros o por el Parlamento Europeo, sino que resulta fijada "desde fuera" por los jefes de Estado y de Gobierno de los Estados miembros a través de tratados, ratificados por los parlamentos o por los ciudadanos a nivel nacional. Los Estados eran en el Tratado Europeo del Carbón y del Acero, en la Comunidad Europea, en la Unión Europea y son ahora en el Tratado constitucional sin lugar a dudas portadores de la iniciativa. No obstante, sería erróneo comprenderlos solo como los primeros impulsores que después de cada celebración de un tratado se retiran y dejan su obra bajo la única responsabilidad de las instituciones y los órganos y por lo tanto conceden a la nueva unidad autonomía e independencia. Muestra la diferencia estructural con el Estado federal, justo el hecho de que los Estados, también después de haber aprobado los tratados constitutivos como firmantes de los mismos, siguen estando presentes y dispuestos a actuar en forma de Conferencia Intergubernamental cuando se trata de modificar dichos Tratados ${ }^{33}$.

La Unión Europea no es, como se ha indicado, un Estado federal clásico, sino una federación de diferente tipo, caracterizada porque las pautas de legitimación y de fuerza vienen desde los Estados singulares. Éstos son y permanecen como partes del tratado y sustentadores de la federación y no sólo como (Estados) miembros. Puesto que una federación no se desgaja de la constelación de origen y no se acoge sólo a la legitimación del pueblo europeo (si existiera) o de los pueblos europeos, su constitución y su desarrollo tampoco se ha desprendido de estos Estados. En la federación, el Consejo de Jefes de Estado y de Gobierno nunca aparece completamente como un órgano de la Unión Europea, es siempre una pieza "exterior". Esto refleja sólo la importante circunstancia de que la Unión Europea adquiere legitimación no sólo desde

32 DIETER GRIMM, "Die grosste Erfindung unserer Zeit", FAZ 16 junio 2003, "Europa an der Schwelle zur Verfassung?", FAZ 16, junio 2003, "Die Verfassung im Prozess der Entstaatlichung" Festschrift Peter Badura (nota 9), pp. 145, 165 ss; también, ¿Braucht Europa eine Verfassung?, 1995.

33 Se puede decir también: en cada modificación constitucional los Estados miembros no sólo actúan como miembros de la Unión Europea sino también como sus fundadores y sustentadores externos. 
abajo, de los pueblos o incluso de su pueblo, sino que, a la vista de la gran distancia entre ciudadanas y ciudadanos y los órganos de la Unión Europea, tal legitimación queda encomendada a los Estados como mediadores. El Tratado Constitucional conceptualiza esto básicamente como doble legitimidad democrática en la Unión Europea.

\section{III.5. Poder Constituyente y Unión EuROPeA}

El Tratado Constitucional de la Unión Europea constituye una Unión que no dispone de un mito fundacional, cuyo origen no está en hechos heroicos dignos de memoria o en cesuras equivalentes ${ }^{34}$. La Constitución configura una unidad nacida de la razón y de reflexiones sobre objetivos, con instituciones y principios racionales y con capacidad de progresar. En la línea del "giro cultural" de las ciencias sociales se ha acentuado mucho la antigua observación de que en la cuna de los Estados nacionales y en la formación de la nación están grandes cesuras, a menudo sangrientas revueltas o revoluciones, y que la escenificación y la posibilidad de rememorar este comienzo sirve para la invención de las propias naciones y su posterior cultura del recuerdo. La descripción histórica es acertada. Por el contrario, no se puede afirmar que también para el futuro pueda deducirse una suerte de ley por la que sólo tales comienzos, con sus sacrificios, puedan servir de base para una fuerte unidad política. Menos convincente aún es pretender que la falta de tales acontecimientos y de tales sacrificios deba considerarse como una "tara de nacimiento" con efectos duraderos. De todos modos cabe afirmar que la Unión Europea "Sólo" es una unidad nacida de la razón y de los intereses con un estatuto fundamental racional. El desarrollo posterior se convierte en un test sobre si una nueva unidad, que se ha producido históricamente sin revolución ni derramamiento de sangre, puede sobrevivir a largo plazo.

\section{III.6. LA PRIMACÍA EN LA UE}

El Derecho originario de la Unión Europea goza de cierta primacía, pero en absoluto de una forma tan sobresaliente como en los Estados. La Ley Fundamental debe comprenderse en particular medida como ordenamiento dotado de primacía, lo que significa que la Constitución es el parámetro para el control del resto de las normas, el Tribunal Constitucional asegura de forma muy efec-

34 El significado especial de surgimiento de la Constitución como un momento histórico excepcional ha sido una y otra vez objeto de la obra de Hasso Hoffmann; al respecto también hace notar que en la discusión europea el concepto de Constitución se desprende del mito del poder constituyente: "Vom Wesen der Verfassung", JöR2003, p. 3 - Para el contexto europeo lo ha recordado recientemente (de modo un tanto radical), adoptando la perspectiva de las ciencias de la cultura ULRICH HALTERN: "Gestalt und Finalität, en A. Von Bogdandy (nota 20), pp. 803, 815 ss. 
tiva este control y garantiza así la función de la Constitución como criterio de medida ${ }^{35}$. ¿Funciona esto de igual modo en el Tribunal de Justicia de las Comunidades Europeas y se le debe comprender como el Tribunal Constitucional de la Unión? ${ }^{36}$ Ciertamente hay casos en los que el TJCE ha ejercido el control sobre los órganos de la Unión Europea, particularmente frente al Consejo y frente al Parlamento, y ha impuesto el Derecho primario como parámetro de validez $^{37}$. El ejemplo más reciente lo podemos encontrar en la cuasi-demanda entre órganos, la Comisión contra el Consejo, en el asunto del pacto de estabilidad, en el que el TJCE ha fallado contra éste en la cuestión procedimental ${ }^{38}$. ¿Pero es este control sobre los órganos la forma de control dominante del TJCE? ¿Y cuando se habla del rango y la primacía en la UE, se afirma en primera línea la relación entre el Derecho primario y el Derecho secundario establecido por los órganos europeos?

La primacía, en relación con la UE, sólo se alcanzará a comprender exactamente cuando se diferencien de forma estricta dos formas de primacía, a saber, la interior o interna, que significa la vinculación del Derecho legal a la primacía del Derecho constitucional interno, y la primacía federal o externa, mediante la cual cualquier esfera de algún modo superior y su Derecho tienen la primacía externa frente al Derecho de las unidades incorporadas. Lo que significa resumidamente: de un lado, la supraordenación interna dentro de la jerarquía normativa de la misma unidad, frente a la primacía de todo el Derecho de la Comunidad superior frente a otra unidad, la integrada. En relación con la UE, esto significa que la primacía de momento casi exclusivamente puede predicarse del ordenamiento jurídico de la UE como primacía federal o externa sobre los ordenamientos de los Estados miembros. El TJCE actúa preferentemente en la esfera externa, en la específica función de integración. Es guardián de la Constitución en primer lugar frente a los Estados miembros, y sólo en segundo lugar como un tribunal constitucional, guardián del Derecho originario frente a los órganos de la UE.

A partir de esta observación inicial se ha observado con razón - cito para abreviar a Armin von Bogdandy ${ }^{39}$ — algo fundamental, a saber, cómo puede peligrar la instauración de lindes horizontales y verticales en la arquitectura insti-

35 RAINER WAHL, "Der Vorrang der Verfassung”, Der Staat 20 (1981), pp. 485 ss; "Konstitutionalisierung - Leitbegriff oder Alterbegriff?, en Libro Homenaje a Brohm, pp. 191 ss.

36 Sobre el papel del TJCE como tribunal constitucional, FRANZ C. MAYER, "Europäische Verfassungsgerichtsbarkeit", en A. V Bogdandy (nota 20) pp. 229 ss. "Wer soll Hüter der europäischen Verfassung sein?", AÖR 129 (2004) pp. 411 ss.; JOSEPH H. H. WEILER, "The transformation of Europe", en id. (ed), The Constitution of Europe, 1999, pp. 10 ss; GIL CARLOS RODRIGUEZ IGLESIAS, "Der Gerichtshof der Europäischen Gemeinschaften als Verfassungsgericht", EuR 1992, pp. 225 ss.

37 Vid especialmente la sentencia del TJCE en el caso C-376/98 sobre la declaración de nulidad de la directiva 98/43/EG del Parlamento Europeo y del Consejo de 6 de julio de 1998 para la armonización de las prescripciones jurídicas y administrativas de los Estados miembros sobre la publicidad y la esposorización del tabaco.

38 TJCE, sentencia de 13 de julio de 2004, Rs C-27/04, NJW2004, 2360

39 ARMIN VON BOGDANDY, "Europäisches Prinzipienrecht", op. cit, (cit.en nota 20), pp. 149 ss., id, "Europäische Verfassung und europäische Identität", JZ 2004, pp. 54 ss. 
tucional conjunta de la UE y los Estados miembros a través de una progresiva política de derechos fundamentales, esto es, también el hacer efectiva la primacía del Derecho primario frente al secundario. Porque una política de derechos fundamentales que busque su reforzamiento podría tener muy notables efectos centralizadores y por lo tanto amenazar el equilibrio político constitucional de la Unión Europea y los Estados miembros ${ }^{40}$.

\section{III.7. El Parlamento Europeo}

Tampoco la forma de gobierno de la Unión Europea ${ }^{41}$ es una repetición del sentido y de la función del sistema parlamentario en el Estado unitario o en el clásico Estado federal. Quien comprenda al Parlamento Europeo como un "todavía no del todo parlamento" o como "parlamento a medias" está colocando el parlamentarismo como medida sin haber verificado su adecuación. Sólo sus funciones como parlamento de trabajo y de control se adecuan a tal perspectiva en la estructura general de la Unión, su función representativa está sin embargo débilmente desarrollada ${ }^{42}$.

\section{DEMOCRACIA Y PRINCIPIO DEMOCRÁTICO: ¿TRANSFERIBILIDAD A LA UNIÓN EUROPEA O ESPECIFICIDAD Y SINGULARIDAD EN LA UNIÓN EUROPEA?}

Un ejemplo clásico de la aquí tratada problemática de la transferibilidad es el principio democrático, sobre el que se ha discutido desde hace mucho tiempo $^{43}$. Muchos ven la cuestión de la democracia de la Unión Europea casi como la prueba de fuego para comprobar si la Unión Europea camina por el sendero correcto o no. Se ha deliberado mucho, no sólo en Alemania sino también en muchos otros Estados miembros, sobre si el principio democrático de los Estados constitucionales nacionales puede ser transferido de forma completa o sólo de manera reducida al plano europeo, y si la actual vida democrática en la UE es deficitaria, es decir, si representa por decirlo así una forma atrofiada de democracia.

40 CHRISTOPH SCHÖNBERGER, "Normenkontrollen im EG-Föderalismus —Die Logik gegenläufiger Hierarchisierungen im Gemeinschaftsrecht, EuR 2003, pp. 600 ss.

41 Sobre ello ahora sobre todo WOLGANG WESSELS, Das politische System der Europäischen Union, 2004.

42 Sobre ello ahora a fondo PHILIPP DANN, "Parlamente in Exekutivföderalismus", Der Staat 42 (2003), pp. 355 ss.

43 Cfr. sobre la discusión en especial GERTUDE LÜBBE-WOLF, "Europäisches und nationales Verfassungsrecht", VVDStRL 60 (2001), pp. 246 ss; Armin von Bogdandy (nota 9), pp. 1033 ss; Marcel Kaufmann, Europäische Integration und Demokratieprinzip, 1997; Winfried Kluth, Die demokratische Legitimation der Europäischen Union, 1995. 
De hecho, de entre todos los principios estructurales político - constitucionales o elementos estructurales, la democracia y el principio democrático deben ser el rasgo decisivo de la configuración política de cualquier unidad política, puesto que expresa lo fundamental sobre los sujetos que son portadores de una comunidad política y la medida de las relaciones internas entre los particulares y la unidad. El Estado de Derecho se puede comprender por ejemplo fácilmente de forma generalizadora e independiente de la configuración concreta de la unidad política. El Estado de Derecho caracteriza así la Organización Mundial de Comercio $(\mathrm{OMC})^{44}$, y el desarrollo de tal postulado puede considerarse como un programa alcanzable también en ese ámbito. Democracia y OMC es por el contrario una combinación que a nadie se le ocurriría de forma espontánea. El problema de la democracia en la Unión Europea es un indicador, entre otros, de la cercanía de la Unión Europea sea a la OMC, sea a los Estados.

\section{IV.1. LA DEMOCRACIA A DOS NIVELES EN LA UNIÓN EUROPEA}

En Alemania, Gertrude Lübbe-Wolf ha defendido de forma expresa y enérgica la completa validez para la UE del principio democrático desarrollado en el ámbito estatal. Ha rechazado de forma explícita el frecuentemente defendido principio de no transferibilidad ${ }^{45}$. En su opinión, la democracia, tal y como se ha desarrollado en el ámbito nacional, debe repetirse en el espacio europeo (y debe crecer en ambas esferas). Lübbe-Wolf expresa así una forma de pensar difundida. Pero pronto, ya en 1964, Peter Badura ${ }^{46}$ formuló la tesis contraria en Alemania. En su opinión, una estructura eficaz de democracia internacional sólo puede realizarse si se especifica que tal realidad en absoluto puede ser construida a partir de la transferencia de las características históricas concretas de la democracia en los ordenamientos constitucionales nacionales a las instituciones internacionales. Debería ser concebida de nuevo a partir de las circunstancias singulares bajo las cuales se funda y se ejerce el poder en el ámbito internacional. Conforme a tal tesis, el principio democrático en la Comunidad Europea adquiere una forma propia y cobra rasgos propios merced al carácter supranacional de esta comunidad. Por ello el principio democrático, como el resto de las decisiones fundamentales del ordenamiento político-constitucional, no pueden referirse solo a la Unión Europea, sino a la relación de la Unión Europea y los Estados miembros. Desde esta óptica, la democracia en la Unión Europea es una democracia compartida y vinculada, por así decirlo una democracia duplicada.

44 MEINHARD HILF, "Die Konstitutionalisierung der Welthandelsordnung: Struktur, Institutionen und Verfahren", en Berichte der Deutschen Gesellschaft für Volkerrrecht, 2003, pp. 257 ss.

45 GERTRUDE LÜBE-WOLFF, (nota 43), pp. 246, 247.

46 PETER BADURA, "Bewahrung und Veränderung demokratischer und rechtsstaatlicher Verfassungsstruktur in den internationalen Gemeinschaften", VVDStRL 23 (1996), pp. 34 ss. 38 y 97 s. Esta tesis de Badura es una pronta y exacta versión de la tesis de la no repetición, puede comprenderse como la melodía de fondo de una larga discusión. 
Puesto que la legitimación del poder en la Unión Europea no puede ser tratada ni pensada de forma única y aislada sólo para la UE, sino que ha de comprender la conexión con la legitimación del poder paralela y complementaria en cada Estado. Así considerado, el problema democrático en la Unión Europea afecta siempre a un vínculo de la UE más el Estado nacional ${ }^{47}$.

La cuestión democrática europea no concierne solo consecuentemente a la forma democrática y a la vida democrática de los europeos sino a los españoles, alemanes, franceses, etc, que conviven en la Europa unida. Ellos viven tanto en su Estado nacional y su democracia, como también en la UE y en su democracia. La promoción de la democracia se orienta por tanto no sólo a la Unión Europea, sino a la constelación general (esto es, el vínculo), y para esta constelación general se debe plantear si existe en conjunto una legitimación democrática suficiente y adecuada. La idea básica del "ordenamiento dividido en dos" deja de ser trivial precisamente cuando cada uno de los ambiciosos conceptos de la teoría del Estado se analiza nuevamente desde la perspectiva de este fenómeno básico de la duplicación. La democracia debe ser en cierto sentido nuevamente "inventada". Debe conceptualizarse y vivirse lo que significa que procesos democráticos recíprocamente relacionados se produzcan tanto en la Unión Europea como en los Estados miembros. No es nuevo el hecho de que la democracia deba ser entendida de forma nueva. Al final del siglo XVIII, la democracia en los grandes Estados territoriales debió ser entendida de forma nueva, después de que por largo tiempo se considerara que sólo era posible en las conocidas y pequeñas ciudades-Estado de la antigüedad ${ }^{48}$. Saltándose esta teoría, los EE.UU mostraron entonces cómo, tanto en cada Estado miembro como en la Unión en su conjunto, la democracia (representativa) era practicable y ejercitable en grandes espacios.

A partir de estos fenómenos básicos de duplicidad mencionados, no puede pasarse por alto que la democracia como categoría básica del orden político no pueda ser simplemente "reduplicada", sino que ambas democracias en la Unión Europea y en los Estados miembros se comporten de forma recíprocamente complementaria y así se modifica la conocida democracia en los Estados. De una parte, cambia necesariamente la estructura democrática interna en cada uno de los Estados miembros de la UE. Esto se evidencia en particular en una pérdida de significación de los parlamentos nacionales ${ }^{49}$. Los ciudadanos

47 Esta consideración se inserta en un amplio abanico de comprensiones estructuralmente similares, que giran todas ellas en torno a la idea de una unión o de un sistema de varios niveles.

48 Jean-Jacques Rousseau está aún tan marcado por la idea de la democracia de asamblea (al mismo tiempo considera necesario un vínculo social fuerte), que considera que su República debe ser un pequeño Estado, mejor una ciudad-Estado; sobre este tema, IRING FETSCHER, Rousseaus politische Philosophie, 1988, pp. 175, 177.

49 Sobre el tema, MATTHIAS HERDERGEN, "Informalisierung und Entparlamentarisierung politischer Entscheidungen als Gefährdungen der Verfassung?", VVDStRL Bd 6 , pp. 9, 11, 26. LoS parlamentos son siempre los perdedores cuando las tareas solo se pueden realizar mediante la cooperación con otros Estados o a través de órganos supranacionales. Toda "política internacional" redunda en pérdidas para el Parlamento. 
individuales en este país experimentan antes o después el hecho de que en Alemania rige no sólo Derecho alemán, sino también Derecho europeo. De otra parte, no puede desarrollarse en el plano supranacional la completa o por así decirlo absorbente fuerza de la democracia mientras que no haya una fuerte base nacional. La política no se desarrolla sólo, ni siquiera principalmente en Bruselas y Estrasburgo; está centrada todavía en primer lugar y de modo dominante en Berlín y París o en un plano regional. Las ciudadanas y los ciudadanos, que a fin de cuentas son decisivos, insisten en tomar en consideración ante todo la política desarrollada en el plano nacional. Justo esta es la diferencia con la arriba citada unidad alemana del siglo xIx. En ella la relevancia política pasó rápidamente a ser la del plano superior y sobre todo la conciencia política de los ciudadanos de ser prusiano o bávaro fue pronto dominada por la de ser alemán ${ }^{50}$. Los procesos de construcción nacional en Alemania y de integración actual en Europa se diferencian en la mentalidad y en el sentimiento político. Ciertamente en ambos procesos se da una duplicación en la situación de la conciencia. Pero las estructuras federales son extremadamente elásticas flexibles y muy creativas. No es necesario, por tanto, que en una unión o camino de integración el nuevo nivel superior arrastre todo hacia sí y lo absorba. La integración europea muestra en cambio el proceso contrario; esto es, el fenómeno de que las unidades menores conservan su significado. Por ello puede quedar abierto que esto sirva también para el orden de competencias. En todo caso, en el campo relativamente independiente de las mentalidades — en la medida en que estas mentalidades codeterminan el centro de gravedad del proceso político-el campo de fuerzas de la política, el ámbito del proceso políti$\mathrm{co}$, el discurso político y las fuerzas de unión siguen manteniéndose como antes en los Estados ${ }^{51}$.

\section{IV.2. La Comunidad Política COMO Fundamento De la DEMOCRACia}

La democracia es por lo demás algo más que la apertura de posibilidades de elección a través del ejercicio del derecho de voto para todos y más que la trabazón de cadenas de legitimación. El éxito vital de la democracia procede esencialmente de líneas de fuerza políticas y de la primaria fuente de poder que supone el sentimiento de pertenencia. El punto de Arquímedes para resolver el tema del déficit democrático europeo es la cuestión a veces ocultada de la comunidad política. Se trata por lo tanto de buscar lo que hace a una unidad po-

50 En el siglo xix hubo una fórmula que hoy, traducida, sería inconcebible para cualquier Estado miembro de la Unión Europea: Prusia queda absorbida por Alemania (cuando Federico Guillermo IV la pronunció en Marzo de 1848 no era sincero; pero posteriormente caló en la conciencia de los ciudadanos de Prusia, en menor medida en las elites).

51 En un cierto grado así vivimos hoy y ciertamente en un futuro próximo hasta que las competencias y las políticas tornen al plano europeo y que la conciencia fundadora y la mentalidad de las ciudadanas y ciudadanos se dirijan a la Unión Europea y asuman este proceso como propio. 
lítica ser tal, y de qué constituye la fuerza de esa unidad política —o mejor: la fuerza de la democracia que allí es posible y se practica. A esta cuestión desatendida durante largo tiempo por la doctrina ${ }^{52}$ dedicaré mis próximas reflexiones; a la Unión Europea como comunidad política, esto es, a las condiciones para la formación de una comunidad no nacional o un orden político simultáneamente nacional y no nacional.

La democracia es una forma de poder que vive de energías y de líneas de fuerza políticas. La democracia significa y promete que la legitimación y las fuentes del poder surgen de abajo, del pueblo. La democracia comprende las líneas de fuerza políticas decisivas, en el sentido de las relaciones recíprocas entre "arriba" y "abajo". Pero lo decisivo es que la democracia comprende y constituye fuerzas reales y sólo puede llevar a la "constitución" lo que ya existe como fuerza política real. La Constitución constituye al Estado completamente y sin excepción conforme a una fórmula destacada del Derecho político y constitucional de la República Federal. Pero lo que la Constitución no constituye y no puede constituir es la voluntad de muchos de ser o no ser una comunidad política. La Constitución no puede, por sí sola o situándose en primer plano, producir aquello que convierte a un grupo de personas en miembros de una Comunidad política. El patriotismo constitucional es un factor de integración; no sirve como elemento único de formación de la comunidad, pues en caso contrario, presupuesta la vigencia de la Constitución, cabría formar Estados o unidades políticas con cualesquiera delimitaciones espacio-geográficas, more geométrico.

Por eso sigue presente la pregunta: ¿Qué convierte una unidad política no estatal en tal unidad? No pueden ser ideas y razonamientos en torno a los derechos del hombre, pues de ellos no trae causa ninguna entidad territorial delimitada y singularizada. Las fuerzas formadoras de una comunidad en la UE pueden ser las que se alimentan, tanto de aspiraciones nacionales como de ideas sobre los derechos humanos. Una tesis difundida opta también por la constelación postnacional (Jürgen Habermas) y por las tendencias observadas de desestatalización. Los deseos y esperanzas unidos a ello, sin embargo, no hacen superflua la cuestión — sino que más bien la fuerzan- sobre qué es lo que, tras suprimir lo nacional, forma, sustenta y mantiene a la Comunidad política en el Estado y, aún mucho más, en la Unión Europea unida.

Subrayar las fuerzas e ideas políticas que están y necesitan estar tras la democracia es una perspectiva inusual para un Derecho público que suele orientarse hacia lo normativo. Necesita por tanto una más detallada fundamentación. A través de la teoría puede intentar comprenderse qué convierte a un grupo en comunidad política. Falta aquí el espacio necesario para hacerlo. En largos periodos históricos puede reconocerse la formación de una comunidad política y su crecimiento de una forma más rápida y evidente. Wolfgang Reinhardt ha

52 Sobre este tema sin embargo, recientemente, MARTÍN NETTESHEIM, "Die politische Gemeinschaft der Unionsbürger", en Festschrift für Peter Häberle, 2004, p. 193. 
expuesto de forma ejemplar y fructífera esta reflexión por así decirlo inductiva en su trabajo "Geschichte der Staatsgewalt" ("Historia del poder estatal"). En su análisis, la perspectiva de los historiadores es simultáneamente la de los politólogos, la de los sociólogos y la de los profesores de Derecho estatal. Interesan tanto las instituciones como los procesos (de formación del poder). La perspectiva histórica abre vía libre a las fuerzas políticas, actores y mentalidades así como a las emociones de quienes intervienen. Si se adopta esta perspectiva, resulta que las cuestiones sobre el surgimiento, la formación, el crecimiento y la fuerza del poder estatal pueden encontrar alguna contestación parcial a través del Derecho que puede mostrar las condiciones marco y de formación. No quedan aquí comprendidas por tanto las auténticas fuerzas formadoras. La democracia (como también el federalismo) precisa de los fundamentos jurídicos, se hace posible por medio de los correspondientes principios jurídico-constitucionales. Pero el que la democracia esté muy arraigada, sea fuerte y se pueda defender frente a sus oponentes en un concreto pueblo y en una específica situación histórica depende evidentemente de otros diversos factores. La cuestión planteada aquí de las fuerzas de formación y movimiento que están detrás de la Unión Europea no es así una cuestión primeramente jurídica. Sin embargo, es una cuestión decisiva para el progreso y la profundización de la integración europea. Quien se interese por si la UE es una comunidad política, una fuerte comunidad política, no puede dirigir la mirada solo y en primera línea a las reglas jurídicas. La cuestión puede ser difícil de responder con rigor metodológico, sobre todo por juristas. Sigue siendo no obstante como una cuestión real y apremiante del orden del día.

El tratamiento en los órdenes federales de la democracia y la cuestión de la unidad política decisiva depende siempre de cuál de los dos planos es al final el determinante. Como fenómeno de conciencia y de valoración juegan un papel los modelos de interpretación subyacentes, por ejemplo si se interpreta la relación entre la Unión Europea y los Estados miembros como una relación entre la base y la superestructura o como una relación del todo con sus partes. Después de todo lo que puede observarse, no parece que los europeos de los diferentes Estados miembros se vuelvan a la Unión Europea como personificación del todo y contemplen su Estado como una mera parte de ese orden. En su lugar parece prevalecer la idea de que sobre la base de los Estados se alza una "Superestructura" en Bruselas y Estrasburgo. Estas someras reflexiones no ponen en duda que en el futuro sean posibles e incuso probables desarrollos que en la Unión Europea pueda crecer un sentimiento colectivo e individual de pertenencia. Está abierta la cuestión de si sus fuerzas serían comparables a las de un Estado nacional; ninguna teoría puede ofrecer en este sentido un pronóstico fundado. Probablemente se desarrollará en la UE una forma más débil de vinculación comunitaria. Tendrá que asumirse en suma la Unión Europea como régimen de débil vínculo y de débil democracia ${ }^{53}$. Pero en este punto

53 Así se expresa otra vez la tesis de que la democracia no es sólo la realización mayor o menor de una forma característica (nacional), sino que hay diferentes modelos de democracia limitados a cada unidad política y adecuados a ella. 
central de las reflexiones puede aprovecharse de nuevo el concepto básico de doble nivel. La democracia de la Unión Europea es importante en sí pero en una UE que comprende la liga de Estados es solo una parte del problema general de la democracia en la UE. Se trata por lo tanto, si se adopta como punto de mira la UE, de la constelación general de la democracia (reducida) en los Estados nacionales más la democracia en la UE.

\section{IV.3. FuERZAS Que FORMAN EUROPA}

¿Cómo se comportan ahora Europa y los ciudadanos de la Unión en sus relaciones recíprocas? Han obtenido una Constitución más o menos en orden, eligen un Parlamento Europeo que dispone de considerables competencias y pueden sentirse seguros de que Europa realiza los mismos buenos valores que los Estados. No obstante, y de modo llamativo, en forma alguna quieren ser europeos, no quieren formar un pueblo europeo ni desarrollar un fuerte sentimiento de pertenencia europea ¿Son por lo tanto todos ellos unos desagradecidos?

Naturalmente cabe mejorar aún la Constitución y conferir al Parlamento aún más derechos. ¿Pero ejercerían entonces la Constitución y la Unión Europea una atracción tan irresistible que las ciudadanas y los ciudadanos se convertirían en europeos convencidos, en ciudadanos de la Unión en el verdadero sentido del término? Algo en este razonamiento falla manifiestamente. Se propone obtener resultados, en concreto el desarrollo de un sentimiento de comunidad y pertenencia; es decir, algo que en 46 años no fue realizable y que a partir de las actuales perspectivas no será alcanzable en los próximos años. ¿De dónde puede proceder esto? Naturalmente se acumulan aquí las respuestas. En primer lugar podría citarse el comienzo tecnocrático - burocrático y la orientación aún importante en tal sentido. ¿Pero son realmente responsables de que no funcionara como muchos pensaban el inicial sendero de desarrollo trazado? ¿Es el sentimiento de pertenencia y de comunidad un proceso "orientado por la oferta"? ¿O acaso hay que tener en cuenta la decisiva "demanda"?

Podría ser que nos hubiéramos equivocado en la comparación entre los siglos XIX y xx. Así se ha divulgado con razón la idea de que las naciones y el sentimiento de nacionalidad del siglo xIx fueron en gran medida una construcción, una invención. Trasladar este pensamiento al siglo xx y comprender la Unión Europea como algo a inventar y construir puede parecer acertado. ¿Pero no se ha desarrollado así una idea correcta hasta sus consecuencias finales que sin embargo podrían ser erróneas? Pues el sentimiento nacional del siglo XIX no fue tan solo una construcción y un descubrimiento. Y sobre todo no parecen bastar tampoco hoy la mera construcción, la política de identidad, ofensivas de aceptación o simplemente un simbólico adorno. Con razón se ha hablado desde hace tiempo en la ciencia histórica en relación con el siglo XIX de fuerzas de formación estatal. Si son válidos los paralelismos y las transferencias de este proceso a la UE, entonces cabe preguntar por las fuerzas de formación europea del 
presente y del futuro. Tales fuerzas son el humus para la democracia europea en la UE y su formación insatisfactoria debilita la democracia. El fenómeno, descrito como déficit democrático, es en definitiva el déficit de tales fuerzas de formación europea y de mentalidades orientadas a Europa en los ciudadanos de la Unión. Si se desarrollaran fuertemente las fuerzas de formación europea en los ciudadanos de la Unión se aproximarían considerablemente las democracias europea y estatal; en caso contrario, la democracia europea y la comprensión de la democracia mantendrían un carácter propio.

Cuando se reconozca de una vez que no hay modelo ni patrón de muestra para la democracia, que el trasfondo teórico de la democracia nacional, europea e internacional no es un concepto democrático cerrado, sino que el tratamiento teórico de la cuestión democrática aconseja centrarse en los distintos niveles específicos, entonces podrán continuarse la reflexión y plantear la cuestión: ¿Está quizá la democracia, enfática y preñada de experiencias, vinculada al Estado y su trasfondo nacional, y por lo tanto no se puede trasladar con facilidad al plano europeo? En la lógica de este razonamiento está también en este caso no considerar imposible la democracia en el plano europeo o tenerla por estructuralmente deficitaria en términos insubsanables; sino reconocer otra forma de democracia para la que es esencial la vinculación con la vida democrática en los Estados miembros. El interés de los individuos, determinante para la vida política, no mengua sólo por la gran extensión de la UE, sino porque no se sienten aún particularmente vinculados a esa gran unidad política. Tiene su propia razón intrínseca que en este caso la vida democrática se articule en dos planos, pero puestos en relación. Esto se corresponde con el carácter de la Unión Europea, que tanto al principio como ahora no puede comprenderse como singularidad, sino sólo en su simultaneidad con los Estados miembros.

\section{REFLEXIÓN FINAL}

La idea final propone de nuevo un amplio trazado: hay una suerte de ley en el desarrollo espiritual conforme a la cual una época, una idea - y aquí puede pensarse también con buenas razones en el Estado constitucional-, en el momento en el que se alcanza su punto culminante y su expresión válida, queda dispuesta para ser disuelta tanto en su núcleo como en sus cimientos por algo novedoso. Mediante el pensamiento puesto en los vínculos y dependencias recíprocas y sobre todo en la coordinación horizontal en vez de en una prevalencia jerárquica ${ }^{54}$, surgen problemas y temas específicos para los cuales deben en primer lugar buscarse los conceptos y las ideas adecuadas, que sólo en parte se han encontrado.

54 La renuncia a la jerarquía y la cuestión de la soberanía afecta directamente incluso al Estado federal, donde realmente ambos conceptos no sirven, pues se legitima un camino que oprime las unidades de abajo. 
El búho de Minerva ha volado hace tiempo sobre los Estados constitucionales nacionales, y ha vuelto otra vez a ellos. Minerva espera aún para hacer volar su búho por una segunda vez en la nueva época, cuando pueda ser comprendida y conceptualizada. En el tiempo entre ambos vuelos debe darse una fase de fantasía, de nuevas propuestas, de desarrollo productivo desde conceptos esenciales de la teoría del Estado hacia conceptos básicos del mundo más allá del Estado, que está todavía vinculado a éste, pero que de igual modo se diferencia de él.

ABstRACT. Rainer Wabl in this research expound if the European Union could be explained throught foundamental principles of State's Theory. At first analysis, he emphasizes the brilliance conceptual of the State's Theory; however in bis opinion is necessary to create new concepts. He refuses the possible comparation of german unification of XIX century with the comunitary integration, and also denies the identity of EU with the classic national State. Moreover Rainer Wabl find the EU as a federation (Bund) where the politic essence is not in the whole Union but in the little Member States. The EU as a federation is based in a main legitimation coming from the State members. The democracy in Europe, although it can be understood as a democracy in several steps, needs a political community that doesn't exist at the present moment. Nevertheless Rainer Wabl believes EU should find new proposals for a scene beyond the State. 